\title{
On Fuzzy Soft Groups
}

\author{
S.V. Manemaran \\ Assistant Professor \\ Department of Mathematics \\ Oxford Engineering College \\ Tiruchirappalli.
}

\begin{abstract}
Molodtsov introduced the concept of soft set theory which can be used as generic mathematical tool for dealing with uncertainty. In this paper, we discuss fuzzy soft sets algebraic structure and give the definition of fuzzy soft group. We define operations on fuzzy soft groups and prove some results on them. Furthermore, definitions of fuzzy soft function and fuzzy soft homomorphism are defined and the theorems on homomorphic image and homomorphic pre image are given. After that, the definition of normal fuzzy soft group is given and some of its basic properties are studied.
\end{abstract}

Keywords: Soft Set, Fuzzy Soft Group, Soft Homomorphism, Normal Fuzzy Soft Group, Pre-image, Fuzzy Soft Function.

\section{INTRODUCTION}

Researchers in economics, engineering, environmental science, sociology, medical science and many other fields deal daily with the complexities of modeling uncertain data. Classical methods are not always successful, because the uncertainties appearing in these domains may be of various types. While Probability theory, fuzzy sets [15], rough sets [12], and other mathematical tools are well-known and often useful approaches to describing uncertainty, each of these theories has its inherent difficulties as pointed out by Molodtsov [7]. Consequently, Molodtsov [7] proposed a completely new approach for modeling vagueness and uncertainly. This is so-called soft theory is free from the difficulties affecting existing methods. In soft set theory the problem of setting the membership function, among other related problems, simply does not arise. This makes the theory very convenient and easy to apply in practice soft set theory has potential applications in many different fields, including the smoothness of functions, game theory, operations research, Riemann integration, Person integration, Probability theory and Measurement theory. Most of these applications have already demonstrated in Molodtsov's book [7]. At present, work on the soft set theory is progressing rapidly. Maji et al [8] describe the application of soft set theory to a decision making problem using rough sets. The same authors have also published a detailed theoretical study on soft sets. The algebraic structure of set theories dealing with uncertainties has also been studied by some authors. Rosenfeld [14] proposed the concept of fuzzy groups in order to establish the algebraic structures of fuzzy sets. Rough groups were defined by Biswas et al [4], and some other author (e.g.,[2] and [8]) have studied the algebraic properties of Rough sets as well. The main purpose of this paper is to introduce a basic version of soft group theory, which extends the notion of a group to include the algebraic structures of soft sets. Our definition of soft groups is similar to the definition of rough groups, but is constructed using different methods. This paper begins by introducing the basic concepts of fuzzy soft set theory then we discuss a basic version of fuzzy soft group theory, which extends the notion of a group to the algebraic structures of fuzzy soft sets. A fuzzy soft group, on the other hand, is a parameterized family of fuzzy subgroups.

\section{PRELIMINARIES}

Definition 2.1: A pair (f,A) is called a soft set over $X$, if $\mathrm{f}: \mathrm{A}: \rightarrow \mathrm{P}(\mathrm{X})$. Here let ' $\mathrm{X}$ ' be the initial Universe and $\mathrm{E}$ be the set of parameters. Let $\mathrm{P}(\mathrm{X})$ denotes the power set $\mathrm{X}$. To illustrate this idea, Molodtsov considered several examples in [7]. Let $\mathrm{I}^{\mathrm{X}}$ denote the set of all fuzzy sets on $\mathrm{X}$.

Definition 2.2: A pair (f,A) is called a soft set over $X$, where $\mathrm{f}: A: \rightarrow I^{\mathrm{x}}\left(\right.$ ie) for each a $\varepsilon A, \quad \mathrm{f}(\mathrm{a})=\mathrm{f}_{\mathrm{a}}: \mathrm{X} \rightarrow \mathrm{I}$ is a fuzzy set in X.

Definition 2.3: $\quad$ Let ' $X$ ' be group and ( $f, A$ ) be a soft set over $\mathrm{X}$, then ( $\mathrm{f}, \mathrm{A}$ ) is said to be a soft group over $\mathrm{X}$ iff $\mathrm{f}(\mathrm{a}) \leq$ $\mathrm{x}, \forall \mathrm{a} \varepsilon$ A. Union of two fuzzy soft sets (f, A) and (g, B) over 
a common Universe $\mathrm{X}$ is the fuzzy soft set $(\mathrm{h}, \mathrm{C})$ where $\mathrm{C}=\mathrm{A}$ $\cup$ B. We write

$(\mathrm{f}, \mathrm{A}) \cup(\mathrm{g}, \mathrm{B})=(\mathrm{h}, \mathrm{C})$. Intersection of two fuzzy soft sets (f, A) and (g, B) over a common Universe $\mathrm{X}$ is the fuzzy soft set $(\mathrm{h}, \mathrm{C})$, where $\mathrm{C}=\mathrm{A} \cap \mathrm{B}$. We write $(\mathrm{f}, \mathrm{A}) \cap(\mathrm{g}, \mathrm{B})=(\mathrm{h}, \mathrm{C})$. Product of two fuzzy soft sets (f, A) and (g, B) denoted (f, A) $\cap(\mathrm{g}, \mathrm{B})$ is defined as $(h, A X B)$ where $h(a, b)=f(a) \cap g(b)$.

Definition 2.4: Let ' $X$ ' be a group and (f, A) be a fuzzy soft set over $\mathrm{X}$, then (f, A) is said to be fuzzy soft group if ;
(i) $\quad \mathrm{f}_{\mathrm{a}}(\mathrm{xy}) \geq \min \left\{\mathrm{f}_{\mathrm{a}}(\mathrm{x}), \mathrm{f}_{\mathrm{a}}(\mathrm{y})\right\}$
(ii) $\quad \mathrm{f}_{\mathrm{a}}\left(\mathrm{x}^{-1}\right) \geq \mathrm{f}_{\mathrm{a}}(\mathrm{x})$
(iii) $\quad \mathrm{f}_{\mathrm{a}}(\mathrm{x})=1 \forall \mathrm{a} \varepsilon \mathrm{A}, \mathrm{x}, \mathrm{y} \varepsilon \mathrm{X}$.

Definiition 2.5: Let ' $f_{a}$ ' be a fuzzy soft set in X.

Let $\phi: \mathrm{X} \rightarrow \mathrm{Y}$ be a map and define $\quad \mathrm{f}_{\mathrm{a}}^{\phi}: \mathrm{X} \rightarrow[0,1]$ by $\mathrm{f}_{\mathrm{a}}^{\phi}(\mathrm{x})=\mathrm{f}_{\mathrm{a}}(\phi(\mathrm{x}), \forall \mathrm{x} \varepsilon \mathrm{X}$.

Definition 2.6: Let (f,A) be a fuzzy soft groups over $\mathrm{X}$ and $\lambda$ $\varepsilon[0,1]$ then

(i) (f, A) is said to be $\lambda$-identity fuzzy soft group over $\mathrm{X}$ if $\mathrm{f}_{\mathrm{a}}(\mathrm{x})=\lambda$ if $\mathrm{x}=\mathrm{e} \forall \mathrm{x} \varepsilon \mathrm{X}$

$=0$ otherwise

(ii) (f, A) is said to be $\lambda$-absolute fuzzy soft group over $X$ if $\mathrm{f}_{\mathrm{a}}(\mathrm{x})=\lambda \forall \mathrm{x} \varepsilon \mathrm{X}, \mathrm{a} \varepsilon \mathrm{A}$.

Definition 2.7: Let (f,A) be a fuzzy soft set over $X$. The soft set $(f, A)_{\alpha}=\left\{\left(f_{a}\right)_{\alpha}: a \varepsilon A\right\}$ for each $\alpha \varepsilon[0,1]$ is called an $\alpha-$ level soft set.

Definition 2.8: Let ' $\mathrm{X}$ ' be a group and $\mathrm{f}_{\mathrm{a}_{1}}, \mathrm{f}_{\mathrm{a}_{2}}, \ldots, \mathrm{f}_{\mathrm{a}_{\mathrm{n}}}$ is n-fuzzy soft subgroups of $\quad X_{1}, X_{2}, \ldots X_{n}$, define a map $\mathrm{f}_{\mathrm{a}_{1}} \chi \mathrm{f}_{\mathrm{a}_{2}} \chi \ldots \mathrm{f}_{\mathrm{a}_{\mathrm{n}}} \chi:\left(\mathrm{X}_{1}, \quad \mathrm{x}_{2}, . . \mathrm{x}_{\mathrm{n}}\right) \rightarrow[0,1]$ by $\left(\mathrm{f}_{\mathrm{a}_{1}} \chi \mathrm{f}_{\mathrm{a}_{2}} \chi \ldots \mathrm{f}_{\mathrm{a}_{\mathrm{n}}} \chi\right) \quad\left(\mathrm{x}_{1}, \mathrm{x}_{2}, \ldots \mathrm{x}_{\mathrm{n}}\right) \quad=\quad \min$ $\left(\mathrm{f}_{\mathrm{a}_{1}}\left(\mathrm{x}_{1}\right) \mathrm{f}_{\mathrm{a}_{2}}\left(\mathrm{x}_{2}\right) \ldots \mathrm{f}_{\mathrm{a}_{\mathrm{n}}}\left(\mathrm{x}_{\mathrm{n}}\right)\right\}$ (this is called generalized product of fuzzy soft groups).

\section{Definition 2.9: Fuzzy Soft group}

Let ' $\mathrm{X}$ ' be a group and ( $\mathrm{f}, \mathrm{A}$ ) be a fuzzy soft set over X. Then (f, A) is said to be fuzzy soft group if (i) $\quad \mathrm{f}_{\mathrm{a}}(\mathrm{xy}) \geq \min \left\{\mathrm{f}_{\mathrm{a}}(\mathrm{x}), \mathrm{f}_{\mathrm{a}}(\mathrm{y})\right\}$

(ii) $\quad \mathrm{f}_{\mathrm{a}}\left(\mathrm{x}^{-1}\right) \geq \mathrm{f}_{\mathrm{a}}(\mathrm{x})$

(iii) $\mathrm{f}_{\mathrm{a}}(\mathrm{x})=1 \forall \mathrm{a} \varepsilon \mathrm{A}, \mathrm{x}, \mathrm{y} \varepsilon \mathrm{X}$.

Throughout this chapter, fuzzy soft set $(f, A)$ is Re called as $f_{a}$.

\section{PROPERTIES OF FUZZY SOFT GROUPS}

Proposition 3.1: Let $f_{a}$ be a fuzzy soft group of $G$ then $f_{a}(x)$ $\leq \mathrm{f}_{\mathrm{a}}(\mathrm{e}) \forall \mathrm{x} \varepsilon$ G. The subset $\quad \mathrm{G}_{\mathrm{fa}}=\left\{\mathrm{x} \varepsilon \mathrm{G} / \mathrm{f}_{\mathrm{a}}(\mathrm{x})=\right.$ $\mathrm{f}(\mathrm{e})\}$ is fuzzy soft group of $\mathrm{G}$.

Proof: Let ' $x$ ' be any element of $G$, then $f_{a}(x y)=\min$ $\left\{\mathrm{f}_{\mathrm{a}}(\mathrm{x}), \mathrm{f}_{\mathrm{a}}(\mathrm{y})\right\}=\min \left\{\mathrm{f}_{\mathrm{a}}(\mathrm{x}), \mathrm{f}_{\mathrm{a}}\left(\mathrm{x}^{-1}\right)\right\} \quad \leq \mathrm{f}_{\mathrm{a}}\left(\mathrm{xx}^{-1}\right)=\mathrm{f}_{\mathrm{a}}(\mathrm{e})$ implies (i). To verify (ii), it follows that $x \in G_{\mathrm{fa}}$ and $\mathrm{G}_{\mathrm{fa}} \neq \phi$.

Now let $\mathrm{x}, \mathrm{y} \varepsilon \mathrm{Gf}_{\mathrm{a}}$.

$\mathrm{f}_{\mathrm{a}}\left(\mathrm{xy}^{-1}\right) \geq \min \left\{\mathrm{f}_{\mathrm{a}}(\mathrm{x}), \mathrm{f}_{\mathrm{a}}\left(\mathrm{y}^{-1}\right)\right\}=\min \left\{\mathrm{f}_{\mathrm{a}}(\mathrm{x}), \mathrm{f}_{\mathrm{a}}(\mathrm{y})\right\}=$ $\min \left\{\mathrm{f}_{\mathrm{a}}(\mathrm{e}), \mathrm{f}_{\mathrm{a}}(\mathrm{e})\right\}=\mathrm{f}_{\mathrm{a}}(\mathrm{e})$

but from (i) $f_{a}\left(x y^{-1}\right) \leq f_{a}(e)$ for all $x, y \varepsilon G$ and so $f_{a}\left(x y^{-1}\right)=$ $\mathrm{f}_{\mathrm{a}}(\mathrm{e})$ which then $\mathrm{xy}^{-1} \varepsilon \mathrm{G}_{\mathrm{fa}}$ is fuzzy soft group of $\mathrm{G}$.

Corollary 3.2: $\quad$ Let ' $G$ ' be a finite group and $f_{a}$ be a fuzzy soft group of $\mathrm{G}$. Consider the subset $\mathrm{H}$ of $\mathrm{G}$ given by $\mathrm{H}$ $=\left\{x \in G / f_{a}(x)=f_{a}(e)\right\}$, then $H$ is a Crisp subgroup of $G$.

Proposition 3.3: Let $\mathrm{f}_{\mathrm{a}}$ and $\mathrm{f}_{\mathrm{b}}$ be two fuzzy soft groups of $\mathrm{G}$, then $f_{a} \cap f_{b}$ is fuzzy soft group of $G$.

Proof: Let $\mathrm{x}, \mathrm{y} \varepsilon \mathrm{G}$.

$(\mathrm{FSG} 1)\left(\mathrm{f}_{\mathrm{a}} \cap \mathrm{f}_{\mathrm{b}}\right)(\mathrm{xy})=\min \left\{\mathrm{f}_{\mathrm{a}}(\mathrm{xy}), \mathrm{f}_{\mathrm{b}}(\mathrm{xy})\right\}$

$\geq \min \left\{\min \left\{\mathrm{f}_{\mathrm{a}}(\mathrm{x}), \mathrm{f}_{\mathrm{a}}(\mathrm{y})\right\}, \min \left(\mathrm{f}_{\mathrm{b}}(\mathrm{x}), \mathrm{f}_{\mathrm{b}}(\mathrm{y})\right\}\right.$

$=\min \left\{\min \left\{\mathrm{f}_{\mathrm{a}}(\mathrm{x}), \mathrm{f}_{\mathrm{b}}(\mathrm{x})\right\}, \min \left\{\mathrm{f}_{\mathrm{a}} \mathrm{y}\right), \mathrm{f}_{\mathrm{b}}(\mathrm{y})\right\}$

$=\min \left\{\left(\mathrm{f}_{\mathrm{a}} \cap \mathrm{f}_{\mathrm{b}}\right)(\mathrm{x}),\left(\mathrm{f}_{\mathrm{a}} \cap \mathrm{f}_{\mathrm{b}}\right)(\mathrm{y})\right\}$

$=\min \left\{\mathrm{f}_{\mathrm{a}}\left(\mathrm{x}^{-1}\right), \mathrm{f}_{\mathrm{b}}\left(\mathrm{x}^{-1}\right)\right\}$

$=\min \left\{f_{a}(x), f_{b}(x)\right\}$

$=\left(f_{a} \cap f_{b}\right)(x)$

Proposition 3.4: If ' $f_{a}$ ' is fuzzy soft group, then the set $\cup\left(f_{a}\right.$; $\mathrm{t})$ is fuzzy soft group $\quad \forall \mathrm{t} \varepsilon \operatorname{Im}\left(\mathrm{f}_{\mathrm{a}}\right)$.

Proof: $\quad$ Let $t \varepsilon \operatorname{Im}(A) \subseteq[0,1], \quad x, y \varepsilon \cup\left(f_{a} ; t\right)$, then $f_{a}(x) \geq t, f_{a}(y) \geq t$. $\quad$ since $f_{a}$ is fuzzy soft group of $G$. 
$\mathrm{f}_{\mathrm{a}}(\mathrm{xy}) \geq \min \left\{\mathrm{f}_{\mathrm{a}}(\mathrm{x}), \mathrm{f}_{\mathrm{a}}(\mathrm{y})\right\} \geq \mathrm{t}$ implies $\quad \mathrm{xy} \varepsilon \cup\left(\mathrm{f}_{\mathrm{a}} ; \mathrm{t}\right)$. Let $\mathrm{x} \varepsilon$

$\cup\left(f_{a} ; t\right)$ implies $\quad f_{a}\left(x^{-1}\right)=f_{a}(x) \geq t$ which gives $x^{-1} \varepsilon \cup\left(f_{a} ; t\right)$.

Thus $\cup\left(f_{a} ; t\right)$ is fuzzy soft group of $G$.

Proposition 3.5: If ' $f_{a}$ ' is fuzzy soft group such that all nonempty level subset $\cup\left(f_{a} ; t\right)$ is fuzzy soft group of $G$, then ' $f_{a}$ ' is fuzzy soft group of G.

Proof: Assume that the non-empty level set $\cup\left(f_{a} ; t\right)$ is fuzzy soft group of $\mathrm{G}$. If $\quad \mathrm{t}_{0}=\min \left\{\mathrm{f}_{\mathrm{a}}(\mathrm{x}), \mathrm{f}_{\mathrm{a}}(\mathrm{y})\right\}$ and for $\mathrm{x}$, $\mathrm{y} \varepsilon \mathrm{G}$, then $\mathrm{x}, \mathrm{y} \varepsilon \cup\left(\mathrm{f}_{\mathrm{a}} ; \mathrm{t}\right)$ so $\mathrm{f}_{\mathrm{a}}(\mathrm{xy}) \geq \mathrm{t}_{0}=\min \left\{\mathrm{f}_{\mathrm{a}}(\mathrm{x})\right.$, $\mathrm{f}_{\mathrm{a}}(\mathrm{y})$ \} which implies that the condition (FSG1) is valid. For $\mathrm{x}^{-1} \varepsilon \mathrm{G}$, then $\quad \mathrm{x}^{-1} \varepsilon \cup\left(\mathrm{f}_{\mathrm{a}} ; \mathrm{t}_{0}\right)$ thus $\mathrm{f}_{\mathrm{a}}\left(\mathrm{x}^{-1}\right)=\mathrm{t}_{0}=\mathrm{f}_{\mathrm{a}}(\mathrm{x})$ which gives the condition (FSG2) is valid and therefore ' $f_{a}$ ' is fuzzy soft group of G.

Proposition 3.6: A set of necessary and sufficient condition for a fuzzy soft set of a group $\mathrm{G}$ to be a fuzzy soft group of $\mathrm{G}$ is that $\mathrm{f}_{\mathrm{a}}(\mathrm{xy}) \geq \min \left\{\mathrm{f}_{\mathrm{a}}(\mathrm{x}), \mathrm{f}_{\mathrm{a}}(\mathrm{y})\right\} \forall \mathrm{x}, \mathrm{y}$ in $\mathrm{G}$.

Proof: Let ' $\mathrm{f}$ ' ' be a fuzzy soft group of $\mathrm{G}$, then

$\mathrm{f}_{\mathrm{a}}\left(\mathrm{xy}^{-1}\right) \geq \min \left\{\mathrm{f}_{\mathrm{a}}(\mathrm{x}), \mathrm{f}_{\mathrm{a}}(\mathrm{y})\right\}$

$=\min \left\{\mathrm{f}_{\mathrm{a}}(\mathrm{x}), \mathrm{f}_{\mathrm{a}}(\mathrm{y})\right\}$ for $\mathrm{x}, \mathrm{y} \varepsilon \mathrm{G}$.

For the Converse part,

Suppose that ' $f_{a}$ ' be fuzzy soft set of the group $G$ of which $\mathrm{e}$ is the identity element.

Now $\mathrm{f}_{\mathrm{a}}\left(\mathrm{yy}^{-1}\right) \geq \min \left\{\mathrm{f}_{\mathrm{a}}(\mathrm{y}), \mathrm{f}_{\mathrm{a}}(\mathrm{y})\right\}$ or

$$
f_{a}(e) \geq f_{a}(y)
$$

Now $\mathrm{f}_{\mathrm{a}}\left(\mathrm{ey}^{-1}\right) \geq \min \left\{\mathrm{f}_{\mathrm{a}}(\mathrm{e}), \mathrm{f}_{\mathrm{a}}(\mathrm{y})\right\}$ or

$$
\begin{gathered}
f_{a}\left(y^{-1}\right) \geq f_{a}(y, e) \\
\text { also, }
\end{gathered}
$$

$\mathrm{f}_{\mathrm{a}}(\mathrm{xy}) \geq \min \left\{\mathrm{f}_{\mathrm{a}}(\mathrm{x}), \mathrm{f}_{\mathrm{a}}(\mathrm{y})\right\} \geq \min \left\{\mathrm{f}_{\mathrm{a}}(\mathrm{x}), \mathrm{f}_{\mathrm{a}}(\mathrm{y})\right\}$.

Proposition 3.7: Let $G$ and $G^{1}$ be two groups and $\theta: G \rightarrow$ $\mathrm{G}^{1}$ be a soft homomorphism. If $\mathrm{f}_{\mathrm{b}}$ is fuzzy soft group of $\mathrm{G}$ then the pre-image $\theta^{-1}\left(f_{b}\right)$ is fuzzy soft group of $G$.

Proof: Assume that $f_{b}$ is fuzzy soft group of $G^{1}$.

Let $\mathrm{x}, \mathrm{y} \varepsilon \mathrm{G}$.

$$
\begin{gathered}
(\mathrm{FSG} 1) \mu_{\theta}^{-1}\left(\mathrm{f}_{\mathrm{b}}\right)(\mathrm{xy}) \quad \mu_{\mathrm{f}_{\mathrm{b}}}(\theta \theta(\mathrm{xy})) \\
=\mu_{\mathrm{f}_{\mathrm{b}}}(\theta(\mathrm{x}) \cdot \theta(\mathrm{y})) \\
\geq \min \left\{\mu _ { \mathrm { f } _ { \mathrm { b } } } \left(\theta(\mathrm{x}), \mu_{\mathrm{f}_{\mathrm{b}}}(\theta(\mathrm{y})\}\right.\right. \\
=\min \left\{\mu_{\theta}^{-1} \mathrm{f}_{\mathrm{b}}(\mathrm{x}), \mu_{\theta}^{-1} \mathrm{f}_{\mathrm{b}}(\mathrm{y})\right\} \\
=\mu_{\mathrm{f}_{\mathrm{b}}}\left(\theta\left(\mathrm{x}^{-1}\right)\right) \\
(\mathrm{FSG} 2) \mu_{\theta}^{-1} \mathrm{f}_{\mathrm{b}}\left(\mathrm{x}^{-1}\right) \quad \mu_{\mathrm{f}_{\mathrm{b}}}\left(\theta^{-1} \mathrm{x}^{-1}=\mu_{\mathrm{f}_{\mathrm{b}}}(\theta \mathrm{x})\right. \\
=\mu_{\theta}^{-1} \mathrm{f}_{\mathrm{b}}(\mathrm{x})
\end{gathered}
$$

$\therefore \quad \theta^{-1}\left(f_{b}\right)$ is fuzzy soft group of G.

Proposition 3.8: Let $\theta: G \rightarrow G^{1}$ be an epimorphism and $f_{b}$ be fuzzy soft set in $G^{1}$. If $\theta^{-1}\left(f_{b}\right)$ is fuzzy soft group of $\mathrm{G}, \mathrm{f}_{\mathrm{b}}$ is fuzzy soft group of $\mathrm{G}^{1}$.

Proof: Let $x, y \varepsilon G^{1}$, then there exists $a, b \varepsilon G$ such that $\theta(a)=x, \theta(b)=y$.

It follows that,

(FSG1) $\mu_{\mathrm{f}_{\mathrm{b}}}(\mathrm{xy})=\mu_{\mathrm{f}_{\mathrm{b}}}(\theta(\mathrm{a}) \theta(\mathrm{b}))$

$$
=\mu_{\mathrm{f}_{\mathrm{b}}}(\theta(\mathrm{ab})
$$

$$
=\mu_{\theta}^{-1} \mathrm{f}_{\mathrm{b}}(\mathrm{ab})
$$

$$
\begin{aligned}
& \geq \min \left\{\mu_{\theta}{ }^{-1} \mathrm{f}_{\mathrm{b}}(\mathrm{a}), \mu_{\theta}{ }^{-1} \mathrm{f}_{\mathrm{b}}(\mathrm{b})\right\} \\
& \geq \min \left\{\mu _ { \mathrm { f } _ { \mathrm { b } } } \left(\theta(\mathrm{a}), \mu_{\mathrm{f}_{\mathrm{b}}}(\theta(\mathrm{b})\}\right.\right. \\
& \geq \min \left\{\mu_{\mathrm{f}_{\mathrm{b}}}(\mathrm{x}), \mu_{\mathrm{f}_{\mathrm{b}}}(\mathrm{y})\right\}
\end{aligned}
$$

(FSG2) $\mu_{\mathrm{f}_{\mathrm{b}}}\left(\mathrm{x}^{-1}\right)=\mu\left(\theta\left(\mathrm{a}^{-1}\right)\right)=\mu_{\mathrm{f}_{\mathrm{b}}}\left(\theta\left(\mathrm{a}^{-1}\right)=\mu_{\theta}^{-1} \mathrm{f}_{\mathrm{b}}\left(\mathrm{a}^{-1}\right)\right.$

Definition 2.10: Let $f_{a}$ be a fuzzy soft set in G. Let $\theta: G \rightarrow$ $G$ be a map and define $\mathrm{f}_{\mathrm{A}}{ }^{\theta}=\mathrm{G} \rightarrow[0,1]$ by $\mathrm{f}_{\mathrm{A}}{ }^{\theta}(\mathrm{x})=$ $f_{A}(\theta(x))$. 
Proposition 3.9: If ' $f$ ' is fuzzy soft group of $G$ and $\theta: G$ $\rightarrow \mathrm{G}^{1}$ be a soft homomorphism of $\mathrm{G}$, then the fuzzy soft set $f_{a}^{\theta}=\left\{x, f_{a}^{\theta}(x) / x \varepsilon G\right\}$ is fuzzy soft group of $G$.

Proof: Let $x, y \in G$, then

(FSG1) $f_{a}^{\theta}(x y) \quad=f_{a}(\theta(x y)$

$$
=\mathrm{f}_{\mathrm{a}}(\theta(\mathrm{x}) \cdot \theta(\mathrm{y}))
$$$$
\geq \min \left\{\mathrm { f } _ { \mathrm { a } } \left(\theta(\mathrm{x}), \mathrm{f}_{\mathrm{a}}(\theta(\mathrm{y})\}\right.\right.
$$$$
=\min \left\{\mathrm{f}_{\mathrm{a}}^{\theta}(\mathrm{x}), \mathrm{f}_{\mathrm{a}}^{\theta}(\mathrm{y})\right\}
$$

$$
\begin{aligned}
\left(\text { FSG2) } \mathrm{f}_{\mathrm{a}}\left(\mathrm{x}^{-1}\right)\right. & =\mathrm{f}_{\mathrm{a}}\left(\theta\left(\mathrm{x}^{-1}\right)\right) \\
& =\mathrm{f}_{\mathrm{a}}\left(\theta^{-1}(\mathrm{x})\right) \\
& =\mathrm{f}_{\mathrm{a}}^{\theta}(\mathrm{x})
\end{aligned}
$$

Thus $\mathrm{f}_{\mathrm{a}}{ }^{\theta}$ is fuzzy soft group of $\mathrm{G}$.

Proposition 3.10: If ' $f_{a}$ ' is fuzzy soft group of $G$. Let $f_{a}^{+}$be a fuzzy soft set in G defined by $\mathrm{f}_{\mathrm{a}}^{+}(\mathrm{x})=\mathrm{f}_{\mathrm{a}}(\mathrm{x})+1-\mathrm{f}_{\mathrm{a}}(\mathrm{e}) \forall \mathrm{x} \varepsilon$ $\mathrm{G}$, then $\mathrm{f}_{\mathrm{a}}^{+}$is normal fuzzy soft group of $\mathrm{G}$ which contains $\mathrm{f}_{\mathrm{a}}$.

Proof: For any $x, y \in G$, it follows that

$$
\begin{array}{r}
\mathrm{f}_{\mathrm{a}}^{+}(\mathrm{x}) \quad=\mathrm{f}_{\mathrm{a}}(\mathrm{x})+1-\mathrm{f}_{\mathrm{a}}(\mathrm{e})=1 . \\
\begin{array}{r}
\text { FSG1 } \mathrm{f}_{\mathrm{a}}^{+}(\mathrm{xy}) \quad=\mathrm{f}_{\mathrm{a}}(\mathrm{xy})+1-\mathrm{f}_{\mathrm{a}}(\mathrm{e}) \\
\geq \min \left\{\mathrm{f}_{\mathrm{a}}(\mathrm{x}), \mathrm{f}_{\mathrm{a}}(\mathrm{y})\right\}+1-\mathrm{f}_{\mathrm{a}}(\mathrm{e}) \\
=\min \left\{\mathrm{f}_{\mathrm{a}}(\mathrm{x})+1-\mathrm{f}_{\mathrm{a}}(\mathrm{e}), \mathrm{f}_{\mathrm{a}}(\mathrm{y})+1-\mathrm{f}_{\mathrm{a}}(\mathrm{e})\right\} \\
=\min \left\{\mathrm{f}_{\mathrm{a}}^{+}(\mathrm{x}) \mathrm{f}_{\mathrm{a}}^{+}(\mathrm{y})\right\} \\
\left(\text { FSG1) } \mathrm{f}_{\mathrm{a}}^{+}\left(\mathrm{x}^{-1}\right) \quad=\mathrm{f}_{\mathrm{a}}\left(\mathrm{x}^{-1}\right)+1-\mathrm{f}_{\mathrm{a}}(\mathrm{e})\right. \\
=\mathrm{f}_{\mathrm{a}}(\mathrm{x})+1-\mathrm{f}_{\mathrm{a}}(\mathrm{e}) \\
=\mathrm{f}_{\mathrm{a}}^{+}(\mathrm{x})
\end{array}
\end{array}
$$

Hence $f_{a}^{+}(x)$ is normal fuzzy soft group of G. Clearly $f_{a} \subseteq f_{a}^{+}$.

Proposition 3.11: If $\left\{\mathrm{f}_{\mathrm{a}_{\mathrm{i}}}\right\}_{\mathrm{i} \varepsilon \text { fa }}$ is a family of fuzzy soft groups of $\mathrm{G}$, then $\cap \mathrm{f}_{\mathrm{a}_{\mathrm{i}}}$ is fuzzy soft group $\mathrm{G}$ whose $\cap \mathrm{f}_{\mathrm{a}_{\mathrm{i}}}=\left\{\left(\mathrm{x}, \wedge \mathrm{f}_{\mathrm{a}_{\mathrm{i}}}(\mathrm{x}) / \mathrm{x} \varepsilon \mathrm{G}\right\}\right.$, where i $\varepsilon \mathrm{f}_{\mathrm{a}}$.

Proof: Let $\mathrm{x}, \mathrm{y} \varepsilon \mathrm{G}$, then for $\mathrm{i} \varepsilon \mathrm{f}_{\mathrm{a}}$, it follows that

$$
\begin{aligned}
& (F S G 1) \cap \mathrm{f}_{\mathrm{a}_{\mathrm{i}}}(\mathrm{xy})=\wedge \mathrm{f}_{\mathrm{a}_{\mathrm{i}}}(\mathrm{xy}) \\
& \geq \wedge \min \left\{\mathrm{f}_{\mathrm{a}_{\mathrm{i}}}(\mathrm{x}), \mathrm{f}_{\mathrm{a}_{\mathrm{i}}}(\mathrm{y})\right\} \\
& =\min \left\{\left(\wedge \mathrm{f}_{\mathrm{a}_{\mathrm{i}}}(\mathrm{x})\right),\left(\wedge \mathrm{f}_{\mathrm{a}_{\mathrm{i}}}(\mathrm{y})\right\}\right. \\
& =\min \left\{\left(\cap \mathrm{f}_{\mathrm{a}_{\mathrm{i}}}\right)(\mathrm{x}),\left(\cap \mathrm{f}_{\mathrm{a}_{\mathrm{i}}}\right)(\mathrm{y})\right\} \\
& (\mathrm{FSG} 2) \operatorname{Let} \mathrm{x} \varepsilon \mathrm{G} \cdot \text { For } \mathrm{i} \varepsilon \mathrm{f}_{\mathrm{a}}, \text { it gives that } \\
& \cap \mathrm{f}_{\mathrm{a}_{\mathrm{i}}}\left(\mathrm{x}^{-1}\right)=\wedge \mathrm{f}_{\mathrm{a}_{\mathrm{i}}}\left(\mathrm{x}^{-1}\right) \\
& \geq \wedge \mathrm{f}_{\mathrm{a}_{\mathrm{i}}}(\mathrm{x}) \\
& \quad=\left(\cap \mathrm{f}_{\mathrm{a}_{\mathrm{i}}}\right)(\mathrm{x})
\end{aligned}
$$

Hence $\left(\cap f_{a_{i}}\right)$ is fuzzy soft group of $G$.

Proposition 3.12: If ' $f a$ ' is fuzzy soft group of $G$, then $f_{a}^{c}$ is fuzzy soft group of G.

$$
\begin{aligned}
& \text { Proof: } \quad \text { Let } x, y \varepsilon G . \\
& \begin{aligned}
\left(\text { FSG1) } \mathrm{f}_{\mathrm{a}}^{\mathrm{c}}(\mathrm{xy})=\right. & 1-\mathrm{f}_{\mathrm{a}}(\mathrm{xy}) \\
& \leq 1-\min \left\{\mathrm{f}_{\mathrm{a}}(\mathrm{x}), \mathrm{f}_{\mathrm{a}}(\mathrm{y})\right\} \\
& =\max \left\{1-\mathrm{f}_{\mathrm{a}}(\mathrm{x}), 1-\mathrm{f}_{\mathrm{a}}(\mathrm{y})\right\} \\
& =\max \left\{\mathrm{f}_{\mathrm{a}}^{\mathrm{c}}(\mathrm{x}), \mathrm{f}_{\mathrm{a}}^{\mathrm{c}}(\mathrm{y})\right\}
\end{aligned} \\
& \left(\begin{array}{rl}
\text { FSG2) } \mathrm{f}_{\mathrm{a}}^{\mathrm{c}}\left(\mathrm{x}^{-1}\right)=1-\mathrm{f}_{\mathrm{a}}\left(\mathrm{x}^{-1}\right) \\
\end{array}\right. \\
& =1-\mathrm{f}_{\mathrm{a}}(\mathrm{x})=\mathrm{f}_{\mathrm{a}}^{\mathrm{c}}(\mathrm{x}) \\
& \left(\text { FSG3) } \mathrm{f}_{\mathrm{a}}^{\mathrm{c}}(\mathrm{e})=1-\mathrm{f}_{\mathrm{a}}(\mathrm{e})=1-1=0\right.
\end{aligned}
$$

Proposition 3.13: Let $f_{a}$ and $f_{b}$ be two fuzzy soft groups of $G$ then $f_{a} \cup f_{b}$ is fuzzy soft group of $G$.

Proof: Let $\mathrm{x}, \mathrm{y} \varepsilon \mathrm{G}$.

$\left(\right.$ FSG1) $\left(f_{a} \cup f_{b}\right)(x y)=\max \left\{f_{a}(x y), f_{b}(x y)\right\}$

$\geq \max \left\{\min \left\{\mathrm{f}_{\mathrm{a}}(\mathrm{x}), \mathrm{f}_{\mathrm{a}}(\mathrm{y})\right\}, \min \left\{\mathrm{f}_{\mathrm{b}}(\mathrm{x}), \mathrm{f}_{\mathrm{b}}(\mathrm{y})\right\}\right.$

$\geq \min \left\{\max \left\{\mathrm{f}_{\mathrm{a}}(\mathrm{x}), \mathrm{f}_{\mathrm{a}}(\mathrm{y})\right\}, \max \left\{\mathrm{f}_{\mathrm{b}}(\mathrm{x}), \mathrm{f}_{\mathrm{b}}(\mathrm{y})\right\}\right.$

$\geq \min \left\{\max \left\{\mathrm{f}_{\mathrm{a}}(\mathrm{x}), \mathrm{f}_{\mathrm{b}}(\mathrm{x})\right\}, \max \left\{\mathrm{f}_{\mathrm{a}}(\mathrm{y}), \mathrm{f}_{\mathrm{b}}(\mathrm{y})\right\}\right.$ 
$\geq \min \left\{\left(f_{a} \cup f_{b}\right)(x),\left(f_{a} \cup f_{b}\right)(y)\right\}$

$\left(\right.$ FSG1) $\left(\mathrm{f}_{\mathrm{a}} \cup \mathrm{f}_{\mathrm{b}}\right)\left(\mathrm{x}^{-1}\right)=\max \left\{\mathrm{f}_{\mathrm{a}}\left(\mathrm{x}^{-1}\right), \mathrm{f}_{\mathrm{b}}\left(\mathrm{x}^{-1}\right)\right\}$

$$
\begin{aligned}
& =\max \left\{f_{a}(x), f_{b}(x)\right\} \\
& =\left(f_{a} \cup f_{b}\right)(x)
\end{aligned}
$$

$\left(\right.$ FSG3) $\left(\mathrm{f}_{\mathrm{a}} \cup \mathrm{f}_{\mathrm{b}}\right)(\mathrm{e})=\max \left\{\mathrm{f}_{\mathrm{a}}(\mathrm{e}), \mathrm{f}_{\mathrm{b}}(\mathrm{e})\right\}$

$=\max \{1,1\}$

$=1$

Proposition 3.14: Let $f_{a}$ and $f_{b}$ be two fuzzy soft groups of $G$, then $f_{a} \times f_{b}$ is fuzzy soft group of $G$.

Proof: Let $\mathrm{x}, \mathrm{y} \varepsilon \mathrm{G}$.

$(F S G 1)\left(f_{a} \times f_{b}\right)(x y)=\min \left\{f_{a}(x y), f_{b}(x y)\right\}$

$\geq \min \left\{\min \left\{\mathrm{f}_{\mathrm{a}}(\mathrm{x}), \mathrm{f}_{\mathrm{a}}(\mathrm{y})\right\}, \min \left\{\mathrm{f}_{\mathrm{b}}(\mathrm{x}), \mathrm{f}_{\mathrm{b}}(\mathrm{y})\right\}\right\}$

$\geq \min \left\{\min \left\{\mathrm{f}_{\mathrm{a}}(\mathrm{x}), \mathrm{f}_{\mathrm{b}}(\mathrm{x})\right\}, \min \left(\mathrm{f}_{\mathrm{a}}(\mathrm{y}), \mathrm{f}_{\mathrm{b}}(\mathrm{y})\right\}\right\}$

$\geq \min \left\{\left(\mathrm{f}_{\mathrm{a}} \times \mathrm{f}_{\mathrm{b}}\right)(\mathrm{x}),\left(\mathrm{f}_{\mathrm{a}} \times \mathrm{f}_{\mathrm{b}}\right)(\mathrm{y})\right\}$

$\left(\right.$ FSG2) $\left(\mathrm{f}_{\mathrm{a}} \times \mathrm{f}_{\mathrm{b}}\right)\left(\mathrm{x}^{-1}\right)=\min \left\{\mathrm{f}_{\mathrm{a}}\left(\mathrm{x}^{-1}\right), \mathrm{f}_{\mathrm{b}}\left(\mathrm{x}^{-1}\right)\right\}$

$\geq \min \left\{\mathrm{f}_{\mathrm{a}}(\mathrm{x}), \mathrm{f}_{\mathrm{b}}(\mathrm{x})\right\}$

$=\left(f_{a} \times f_{b}\right)(x)$

$($ FSG3 $)\left(f_{a} \times f_{b}\right)(e)=\min \left\{f_{a}(e), f_{b}(e)\right\}$

$=\min \{1,1\}$

$=1$

Proposition 3.15: Let ' $f_{a}$ ' be a fuzzy soft group over $X,(\phi$, $\chi)$ be a fuzzy soft homomorphism from $X$ to $Y$, then $(\phi, \chi) f_{a}$ is a fuzzy soft group over Y.

Proof: Let $\mathrm{k} \varepsilon \chi_{\mathrm{a}}$ and $\mathrm{y}_{1}, \mathrm{y}_{2} \varepsilon$ Y. If $\phi^{-1}\left(\mathrm{y}_{1}\right)=0, \phi^{-1}\left(\mathrm{y}_{2}\right)=0$ the proof is straight forward.

Let us assume that there exist $\mathrm{x}_{1}, \mathrm{x}_{2} \varepsilon \mathrm{X}$ such that $\phi\left(\mathrm{x}_{1}\right)=\mathrm{y}_{1}, \phi\left(\mathrm{x}_{2}\right)=\mathrm{y}_{2}$.

$\phi\left(\mathrm{f}_{\mathrm{k}}\right)\left(\mathrm{y}_{1} \mathrm{y}_{2}^{-1}\right) \quad=$

$\underset{\left(\varphi(f)=y_{1} y_{2}^{-1}\right)(\chi(a)=k)}{V} f_{a}(t)$ $\geq \underset{(\chi(\mathrm{a})=\mathrm{k})}{\vee} \mathrm{f}_{\mathrm{a}}\left(x_{1}, x_{2}^{-1}\right)$

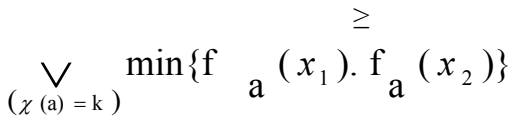

This is equality is satisfied for each

$\mathrm{x}_{1}, \mathrm{x}_{2} \varepsilon X$, which satisfy $\phi\left(\mathrm{x}_{1}\right)=\mathrm{y}_{1}$,

$\phi\left(\mathrm{x}_{2}\right)=\mathrm{y}_{2}$. We have,

$\phi\left(\mathrm{f}_{\mathrm{k}}\right)\left(\mathrm{y}_{1} \mathrm{y}_{2}^{-1}\right)$

$=\min \left\{\underset{\left(\varphi\left(f_{1}\right)=y_{1}\right)}{\vee} \underset{(\chi(\mathrm{a})=\mathrm{k})}{\mathrm{V}} \mathrm{f}^{\mathrm{a}}\left(\mathrm{t}_{1}\right), \underset{\left(\varphi\left(\mathrm{f}_{2}\right)=\mathrm{y}_{2}\right)}{\mathrm{V}(\chi(\mathrm{a})=\mathrm{k})} \underset{\mathrm{a}}{\mathrm{f}}(\mathrm{t} 2)\right\}$

$=\min \left\{\phi(\mathrm{f})_{\mathrm{k}}\left(\mathrm{y}_{1}\right) \cdot \phi(\mathrm{f})_{\mathrm{k}}\left(\mathrm{y}_{2}\right)\right\}$

Proposition 3.16: Let $g_{b}$ be a fuzzy soft group over $Y$ and $(\phi$, $\chi)$ be a fuzzy soft homomorphism from $X$ to $Y$, then $(\phi, \chi)^{-1} g_{b}$ is a fuzzy soft group over X.

Proof: Let a $\varepsilon \chi^{-1}{ }_{\mathrm{b}}$ and $\mathrm{x}_{1}, \mathrm{x}_{2} \varepsilon \mathrm{X}$.

$$
\begin{aligned}
& \phi^{-1}(\mathrm{~g})_{\mathrm{a}}\left(\mathrm{x}_{1} \cdot \mathrm{x}_{2}^{-1}\right) \quad=\mathrm{g}_{\chi(\mathrm{a})}\left(\phi \mathrm{x}_{1} \mathrm{x}_{2}^{-1}\right) \\
& =\mathrm{g}_{\chi(\mathrm{a})}\left\{\phi\left(\mathrm{x}_{1}\right) \cdot \phi\left(\mathrm{x}_{2}\right)\right\} \\
& \geq \min \left\{\mathrm{g}_{\chi(\mathrm{a})}\left(\phi\left(\mathrm{x}_{1}\right)\right) \cdot \mathrm{g}_{\chi(\mathrm{a})}\left(\left(\mathrm{x}_{1}\right)\right)\right\} \\
& =\min \left\{\phi^{-1}(\mathrm{~g})_{\mathrm{a}}\left(\mathrm{x}_{1}\right), \phi^{-1}(\mathrm{~g})_{\mathrm{a}}\left(\mathrm{x}_{2}\right)\right\}
\end{aligned}
$$

Proposition 3.17: Let ' $f_{a}$ ' be a fuzzy soft set over $X$, then ' $\mathrm{f}_{\mathrm{a}}$ ' is a fuzzy soft group over $\mathrm{X}$ iff $\forall \mathrm{a} \varepsilon \mathrm{A}$ and for arbitrary $\alpha$ $\varepsilon[0,1]$ with $(\mathrm{fa})_{\alpha} \neq 0$, the $\alpha$ - level soft set $\left(\mathrm{f}_{\mathrm{a}}\right)_{\alpha}$ is a fuzzy soft group over $\mathrm{X}$.

Proof: Let ' $\mathrm{a}_{\mathrm{a}}$ ' be a fuzzy soft group over $\mathrm{X}$, then for each a $\varepsilon A, f_{a}$ is a fuzzy subgroup of $X$. For arbitrary $\alpha \varepsilon[0,1]$ with (fa) $)_{\alpha} \neq 0$.

Let $x, y \varepsilon\left(f_{a}\right)_{\alpha}$, then $f_{a}(x) \geq \alpha$ and $f_{a}(y) \geq \alpha$.

$\therefore\left(\right.$ FSG1) ${ }_{a}(\mathrm{xy}) \geq \min \left\{\mathrm{f}_{\mathrm{a}}(\mathrm{x}), \mathrm{f}_{\mathrm{a}}(\mathrm{y})\right\}$

$$
\geq \min \{\alpha, \alpha\}=\alpha
$$

Hence xy $\varepsilon\left(f_{a}\right) \alpha$.

Furthermore, 
(FSG2) $\quad f_{a}\left(x^{-1}\right) \geq f_{a}(x) \geq \alpha$, then $x^{-1} \varepsilon\left(f_{a}\right)_{\alpha}$.

We obtain that $\left(\mathrm{f}_{\mathrm{a}}\right)_{\alpha}$ is a subgroup of $\mathrm{X}, \forall$ a $\varepsilon \mathrm{A}$, there exists a $\varepsilon$ A such that $f_{a}$ is not a fuzzy sub group of $X$, then there exist $\mathrm{x}_{0}, \mathrm{y}_{0} \varepsilon \mathrm{X}$ such that, $\mathrm{f}_{\mathrm{a}}\left(\mathrm{x}_{0} \mathrm{y}_{0}{ }^{-1}\right) \leq \min \left\{\mathrm{f}_{\mathrm{a}}\left(\mathrm{x}_{0}\right)\right.$, $\left.\mathrm{f}_{\mathrm{a}}\left(\mathrm{y}_{0}{ }^{-1}\right)\right\}$.

$$
\text { Let } \mathrm{f}_{\mathrm{a}}\left(\mathrm{x}_{0} \mathrm{y}_{0}{ }^{-1}\right)=\lambda, \mathrm{f}_{\mathrm{a}}\left(\mathrm{x}_{0}\right)=\beta \text { and } \mathrm{f}_{\mathrm{a}}\left(\mathrm{y}_{0}\right)=\delta \text {. }
$$

We have, $\lambda<\min \{\beta, \delta\}$.

$$
\text { Let } \alpha=\frac{\lambda+\min \{\beta, \delta\}}{2}, \lambda<\alpha<\min \{\beta, \delta\} \text {. }
$$

Now we have, $\mathrm{f}_{\mathrm{a}}\left(\mathrm{x}_{0} \mathrm{y}_{0}{ }^{-1}\right)=\alpha<\alpha$. Hence $\mathrm{x}_{0}, \mathrm{y}_{0}{ }^{-1}$ $\notin\left(\mathrm{f}_{\mathrm{a}}\right)_{\alpha}$. But since $\beta>\min \{\beta, \delta\}$ and $\delta>\min \{\beta, \delta\}>\alpha$. We obtain $\mathrm{f}_{\mathrm{a}}\left(\mathrm{x}_{0}\right)>\alpha$ and $\mathrm{f}_{\mathrm{a}}\left(\mathrm{y}_{0}\right)>\alpha$. (i.e) $\mathrm{x}_{0}, \mathrm{y}_{0} \varepsilon\left(\mathrm{f}_{\mathrm{a}}\right)_{\alpha}$. This contradicts with the fact that $\left(\mathrm{f}_{\mathrm{a}}\right)_{\alpha}$ is fuzzy soft group over X.

Definition 2.11: $\quad$ Let $\phi: \mathrm{X} \rightarrow \mathrm{Y}$ and $\chi: \mathrm{A} \rightarrow \mathrm{B}$ be two functions, where A and B are parameter sets for the crisp sets $\mathrm{X}$ and $\mathrm{Y}$ respectively, then the pair $(\phi, \chi)$ is called a fuzzy soft function from $\mathrm{X}$ to $\mathrm{Y}$.

Proposition 3.18: If $\mathrm{f}_{\mathrm{a}_{1}}, \mathrm{f}_{\mathrm{a}_{2}}, \ldots, \mathrm{f}_{\mathrm{a}_{\mathrm{n}}}$ be fuzzy soft groups of the groups $\chi_{1}, \chi_{2}, \quad . . \quad \chi_{\mathrm{n}}$ respectively, then $\mathrm{f}_{\mathrm{a}_{1}} \chi \mathrm{f}_{\mathrm{a}_{2}} \chi \ldots \mathrm{f}_{\mathrm{a}_{\mathrm{n}}} \chi$ is fuzzy soft groups of $\chi_{1} \times \chi_{2} \times \ldots . . \times \chi_{\mathrm{n}}$

Proof: For all elements $\left(\mathrm{x}_{1} \mathrm{x}_{2} \ldots \ldots \mathrm{x}_{\mathrm{n}}\right),\left(\mathrm{y}_{1} \mathrm{y}_{2} \ldots . . \mathrm{y}_{\mathrm{n}}\right) \varepsilon \chi_{1} \times \chi_{2}$ $\times \ldots . . \times \chi_{n}$

(FSG1) $\left(\mathrm{f}_{\mathrm{a}_{1}} \chi \mathrm{f}_{\mathrm{a}_{2}} \chi \ldots \mathrm{f}_{\mathrm{a}_{\mathrm{n}}} \chi\right)\left(\left(\mathrm{x}_{1} \mathrm{x}_{2} \ldots . . \mathrm{x}_{\mathrm{n}}\right),\left(\mathrm{y}_{1} \mathrm{y}_{2} \ldots . . \mathrm{y}_{\mathrm{n}}\right)\right)$

$=\left(\mathrm{f}_{\mathrm{a}_{1}} \chi \mathrm{f}_{\mathrm{a}_{2}} \chi \ldots \mathrm{f}_{\mathrm{a}_{\mathrm{n}}} \chi\right)\left(\mathrm{x}_{1} \mathrm{y}_{1}, \mathrm{x}_{2} \mathrm{y}_{2}, \ldots \mathrm{x}_{\mathrm{n}} \mathrm{y}_{\mathrm{n}}\right)$

$\geq \min \left\{\mathrm{f}_{\mathrm{a}_{1}}\left(x_{1} y_{1}\right), \mathrm{f}_{\mathrm{a}_{2}}\left(x_{2} y_{2}\right), \ldots \mathrm{f}_{\mathrm{a}_{\mathrm{n}}}\left(x_{n} y_{n}\right)\right\}$

$\geq \min \{\min \{$

$\left.\mathrm{f}_{\mathrm{a}_{1}}\left(x_{1}\right) \mathrm{f}_{\mathrm{a}_{1}}\left(y_{1}\right)\right\} \min \left\{\mathrm{f}\left(x_{2}\right), \mathrm{f}_{\mathrm{a}_{2}}\left(y_{2}\right)\right\} . . \min \left\{\mathrm{f}_{\mathrm{n}}\left(x_{n}\right), \mathrm{f}_{\mathrm{a}_{\mathrm{n}}}\left(y_{n}\right)\right\}$

$\geq \min \{\min \{$

$\left.\left.\left.\mathrm{f}_{\mathrm{a}_{1}}\left(x_{1}\right) \mathrm{f}_{\mathrm{a}_{2}}\left(x_{2}\right) \cdots \mathrm{f}_{\mathrm{a}_{\mathrm{n}}}\left(x_{n}\right)\right\} \min \left\{\underset{\mathrm{a}_{1}}{\mathrm{f}}\left(y_{1}\right)\right\} \mathrm{f}_{\mathrm{a}_{2}}\left(y_{2}\right) \ldots \mathrm{f}_{\mathrm{a}_{\mathrm{n}}}\left(y_{n}\right)\right\}\right\}$ $\geq \quad \min \left\{\quad\left(\left(\mathrm{f}_{\mathrm{a}_{1}} \chi \mathrm{f}_{\mathrm{a}_{2}} \chi \ldots \mathrm{f}_{\mathrm{a}_{\mathrm{n}}} \chi\right) \quad\left(\left(\mathrm{x}_{1} \mathrm{x}_{2} \ldots \ldots \mathrm{x}_{\mathrm{n}}\right)\right.\right.\right.$,

$\left.\left(\mathrm{f}_{\mathrm{a}_{1}} \chi \mathrm{f}_{\mathrm{a}_{2}} \chi \ldots \mathrm{f}_{\mathrm{a}_{\mathrm{n}}} \chi\right)\left(\mathrm{y}_{1} \mathrm{y}_{2} \ldots . . \mathrm{y}_{\mathrm{n}}\right)\right\}$

$(\mathrm{FSG} 2) \quad\left(\mathrm{f}_{\mathrm{a}_{1}} \chi \mathrm{f}_{\mathrm{a}_{2}} \chi \ldots \mathrm{f}_{\mathrm{a}_{\mathrm{n}}} \chi\right)\left(\mathrm{x}_{1}, \mathrm{x}_{2}, \ldots . . \mathrm{x}_{\mathrm{n}}\right)^{-1}$

$=\left(\mathrm{f}_{\mathrm{a}_{1}} \chi \mathrm{f}_{\mathrm{a}_{2}} \chi \ldots \mathrm{f}_{\mathrm{a}_{\mathrm{n}}} \chi\right)\left(\left(\mathrm{x}_{1}^{-1}, \mathrm{x}_{2}^{-1}, \ldots \mathrm{x}_{\mathrm{n}}^{-1}\right)\right.$

$=\min \left\{\mathrm{f}_{\mathrm{a}_{1}}\left(\mathrm{x}_{1}^{-1}\right) \mathrm{f}_{\mathrm{a}_{2}}\left(\mathrm{x}_{2}^{-1}\right) \ldots \mathrm{f}_{\mathrm{a}_{\mathrm{n}}}\left(\mathrm{x}_{\mathrm{n}}^{-1}\right)\right\}$

$\geq \min \left\{\mathrm{f}_{\mathrm{a}_{1}}\left(\mathrm{x}_{1}\right) \mathrm{f}_{\mathrm{a}_{2}}\left(\mathrm{x}_{2}\right) \ldots \mathrm{f}_{\mathrm{a}_{\mathrm{n}}}\left(\mathrm{x}_{\mathrm{n}}\right)\right\}$

$\geq\left(\mathrm{f}_{\mathrm{a}_{1}} \chi \mathrm{f}_{\mathrm{a}_{2}} \chi \ldots \mathrm{f}_{\mathrm{a}_{\mathrm{n}}} \chi\right)\left(\mathrm{x}_{1} \mathrm{x}_{2} \ldots \ldots \mathrm{x}_{\mathrm{n}}\right)$

(FSG3) $\left(\mathrm{f}_{\mathrm{a}_{1}} \chi \mathrm{f}_{\mathrm{a}_{2}} \chi \ldots \mathrm{f}_{\mathrm{a}_{\mathrm{n}}} \chi\right)\left(\mathrm{e}_{1}, \mathrm{e}_{2}, \ldots \mathrm{e}_{\mathrm{n}}\right)$

$=\min \left\{\mathrm{f}_{\mathrm{a}_{1}}\left(\mathrm{e}_{1}\right) \mathrm{f}_{\mathrm{a}_{2}}\left(\mathrm{e}_{2}\right) \ldots \mathrm{f}_{\mathrm{a}_{\mathrm{n}}}\left(\mathrm{e}_{\mathrm{n}}\right)\right\}$

$=\min \{1,1, \ldots \ldots 1\}$

$=1$

\section{CONCLUSION}

In this paper we studied the algebraic properties of fuzzy soft sets in group structures. This work focused on fuzzy soft groups, homomorphism of fuzzy soft groups and pre-image of fuzzy soft groups. To extend this work one could study the properties of fuzzy soft sets in other algebraic structures such as rings, nor-rings, field and ideals.

\section{REFERENCES}

[1] H. Aktas and N. Cagman, Soft sets and soft group, Information Science 177 (2007), PP 2726-2735.

[2] K. Atanasov, "Intuitionistic Fuzzy Sets", Fuzzy Sets and Systems, No.20, PP 87-96, 1986.

[3] K. Atanasov, "Operators over Interval valued Intuitionistic Fuzzy Sets", Fuzzy Sets and Systems, No.64, PP 159174,1994

[4] R. Biswas and S. Nanda, "Rough groups and tough subgroups”, Bull Polish Acad. Math. No.42, 1994.

[5] Iwinski, "Algebraic approach to rough sets", Sci. math. No.35, PP 673-683, 1987.

[6] G.J. Klir and B. Yuan, Fuzzy sets and fuzzy logic theory and applications, Prettice Helltic, New Jersey (1995).

[7] D. Molodtsov, "Soft Set Theory - First Results", Comput. Math. Appl. No.37, PP 19-31, 1999. 
[8] P. K. Maji, R. Bismas and A.R. Roy, "Fuzzy Soft Sets", The Journal of Fuzzy Mathematics, Vol.3, No.9, PP 589602, 2001

[9] H. Prade and D. Dubois, "Fuzzy Sets and System theory and applications", Academic Press London, 1980.

[10] Z. Pololak, "Hard Set and Soft Sets", Ics. Research Report, Institute of Computer Science Poland, 1994.

[11] D. Pei and D. Miao, From soft sets to information systems, Granular computing, 2005 IEEE International conference on (2)(2005), PP 617 -621.
[12] Z. Pawlak, "Rough Sets", Int. J. inform. Comput. Sci. No.11, PP 341- 356, 1982.

[13] A.R. Roy and P.K. Maji, "A Fuzzy Soft Set Theoretic approach to decision making problems", Journal of Computational and Applied Mathematics, No.203, PP 412-418, 2007.

[14] A. Rosenfeld, "Fuzzy Groups", J.Math. Anal. Appl. No.35, PP 512-517, 1971.

[15] L.A. Zadeh, "Fuzzy Sets", Inform \& Control, No.8, PP 338-353, 1965. 